\title{
Superradiant Phenomena for Spinor Fields in Rotating Black Hole Geometry
}

\author{
Masakatsu Kenmoku ${ }^{1, \star}$, Yongmin $\mathrm{Cho}^{2,3, \star \star}$, Kazuyasu Shigemoto ${ }^{4, \star \star \star}$, and Jong-Hyuk \\ Yoon ${ }^{2, \star \star \star \star}$ \\ ${ }^{1}$ Nara Women's University and Nara Science Academy \\ ${ }^{2}$ Konkuk University \\ ${ }^{3}$ Seoul National University \\ ${ }^{4}$ Tezukayama University
}

\begin{abstract}
We derive the results (i) the ortho-normal and completeness relations for normal modes and (ii) non-existence of zero mode for spinor fields in rotating black hole geometry. From these results, we show that superradiant phenomena for spinor fields should be type 2: positive momentum on the horizon $\left(p_{\mathrm{H}}>0\right)$ and negative frequency at infinity $(\omega<0)$.
\end{abstract}

\section{Introduction}

Matter interactions with black holes (BHs) are essential for their existence and observation. As the black hole geometry, rotating BH geometry is important for superradiant phenomena (incident intensity < scattered intensity). Especially Anti-de Sitter (AdS) space-time plays the role of reflection mirror and successive superradiant phenomena lead to instability of BHs. Spinors are fundamental as matter fields because multiple spinors can represent Bosons and Fermions, which can be realized by the Bargmann-Wigner formulation.

The organization of this short report is as follows. In sect. 2, spinor fields in Kerr-AdS space-time is studied. In sect. 3, normal modes are studied to derive the ortho-normal and completeness relations. In sect. 4, non-existence of zero mode $\left(p_{\mathbf{H}}=\omega-\Omega_{\mathbf{H}} m=0\right)$ is shown with $\omega, m$ as frequency and azimuthal quantum number of spinor fiedls, and $\Omega_{\mathrm{H}}$ as angular velocity of $\mathrm{BH}$. In sect. 5 , we show that the type 2 superradiant modes $\left(\omega<0, p_{\mathbf{H}}>0\right)$ are consistent. Summary of the obtained new results is given in the final section.

This report is based on our paper [1] including the detailed logic and calculation.

\footnotetext{
${ }^{\star}$ e-mail: m.kenmoku@cc.nara-wu.ac.jp

$\star \star$ e-mail: ymcho7@konkuk.ac.kr

$\star \star \star$ e-mail: shigemot@tezukayama-u.ac.jp

$\star \star \star \star$ e-mail: yoonjh@konkuk.ac.jp
} 


\section{Spinor fields in Kerr-AdS space-time}

The line element of Kerr-AdS space-time with Boyer-Lindquist coordinate is

$$
d s^{2}=\frac{\Delta_{r}}{\rho^{2}}\left(d t-\frac{a \sin ^{2} \theta}{\Xi} d \phi\right)^{2}+\frac{\Delta_{\theta} \sin ^{2} \theta}{\rho^{2}}\left(a d t-\frac{r^{2}+a^{2}}{\Xi} d \phi\right)^{2} \frac{\rho^{2}}{\Delta_{r}} d r^{2}+\frac{\rho^{2}}{\Delta_{\theta}} d \theta^{2},
$$

with

$$
\begin{aligned}
& \Delta_{r}=\left(r^{2}+a^{2}\right)\left(1+r^{2} \ell^{-2}\right)-2 M r, \Delta_{\theta}=1-a^{2} \ell^{-2} \cos ^{2} \theta, \\
& \rho^{2}=r^{2}+a^{2} \cos ^{2} \theta, \quad \Xi=1-a^{2} \ell^{-2},
\end{aligned}
$$

where $\ell=\sqrt{-3 / \Lambda}$ is the cosmological parameter and $a=J / M$ is the rotation parameters of BH. The event horizon is defined as the outer zero of $\Delta_{r}$ as $0=\Delta_{r} \Rightarrow r=r_{+}$.

The Dirac equation for spinor fields in curved spacetime is defined through local Minkowski spacetime, where the Dirac matrices are treated as spacetime independent (Greek suffix $(\mu, \nu \ldots)$ as curved spacetime and Latin suffix $(\mathrm{i}, \mathrm{j}, \ldots)$ as local Cartesian spacetime):

$$
\begin{aligned}
0 & =\left(\gamma^{\mu}\left(\partial_{\mu}+\frac{1}{4} \omega_{\mu}^{i j} \Gamma_{i j}\right)+\bar{\mu}\right) \Psi(x) \\
& =\left(\gamma^{i} b_{i}^{\mu} \partial_{\mu}+\frac{1}{2 \sqrt{-g}} \gamma^{i} \partial_{\mu}\left(\sqrt{-g} b_{i}^{\mu}\right)+\frac{1}{4} b_{i}^{\mu} b_{j}^{v} \partial_{\mu} b_{v k} \Gamma^{i j k}+\bar{\mu}\right) \Psi(x),
\end{aligned}
$$

where $\omega_{\mu}^{i j}, b_{i}^{\mu}$ are denoted as spin connection, vierbein, $\Gamma_{i j}, \Gamma_{i j k}$ as anti-symmetric product of Dirac gamma matrix, and $\bar{\mu}$ as mass of spinor field.

We put the spinor fields as separation of variables in the polar coordinate:

$$
\Psi(x)=\frac{\exp (-i \omega t+i m \phi)}{\left(\Delta_{r} \Delta_{\theta} \rho^{2} \sin ^{2} \theta\right)^{1 / 4}} F(r, \theta) .
$$

Next we define the chirality eigen-states as

$$
F(r, \theta)=\left(\frac{\chi^{*}}{\chi}\right)^{1 / 4} f_{+}+\left(\frac{\chi}{\chi^{*}}\right)^{1 / 4} f_{-}, \quad \chi=r+i a \cos \theta, \quad \gamma^{5} f_{ \pm}= \pm f_{ \pm},
$$

where four component spinors are expressed in two component ones:

$$
f_{+}(r, \theta)=\frac{1}{\sqrt{2}}\left(\begin{array}{c}
\zeta \\
-\zeta
\end{array}\right), f_{-}(r, \theta)=\frac{1}{\sqrt{2}}\left(\begin{array}{l}
\eta \\
\eta
\end{array}\right)
$$

with

$$
\eta=\left(\begin{array}{l}
R_{1}(r) S_{1}(\theta) \\
R_{2}(r) S_{2}(\theta)
\end{array}\right), \quad \zeta=\left(\begin{array}{l}
R_{2}(r) S_{1}(\theta) \\
R_{1}(r) S_{2}(\theta)
\end{array}\right) .
$$

Then the Dirac equation reduces to the four ordinary differential equations:

$$
\begin{gathered}
\left(\sqrt{\Delta_{\theta}} \partial_{\theta}+\frac{a \omega \sin \theta^{2}-\Xi m}{\sqrt{\Delta_{\theta}} \sin \theta}\right) S_{1}(\theta)=(-a \bar{\mu} \cos \theta+\kappa) S_{2}(\theta), \\
\left(\sqrt{\Delta_{\theta}} \partial_{\theta}-\frac{a \omega \sin \theta^{2}-\Xi m}{\sqrt{\Delta_{\theta}} \sin \theta}\right) S_{2}(\theta)=(-a \bar{\mu} \cos \theta-\kappa) S_{1}(\theta), \\
\left(\sqrt{\Delta_{r}} \partial_{r}-i \frac{\left(r^{2}+a^{2}\right) \omega-a \Xi m}{\sqrt{\Delta_{r}}}\right) R_{1}(r)=(\kappa-i \bar{\mu} r) R_{2}(r), \\
\left(\sqrt{\Delta_{r}} \partial_{r}+i \frac{\left(r^{2}+a^{2}\right) \omega-a \Xi m}{\sqrt{\Delta_{r}}}\right) R_{2}(r)=(\kappa+i \bar{\mu} r) R_{1}(r),
\end{gathered}
$$

where $\kappa$ is the separation parameter. 


\section{Normal modes: orthonormal and completeness relations}

The local conservation law of the bi-field current $k_{\text {spinor }}^{\mu}$ for $\Psi$ and $\Psi^{\prime}$ holds using the Dirac equations:

$$
\partial_{\mu} k_{\text {spinor }}^{\mu}=0, \quad k_{\text {spinor }}^{\mu}=i \sqrt{-g} \bar{\Psi}(t, x) \gamma^{\mu} \Psi^{\prime}(t, x) .
$$

The inner product defining the integral of $k_{\text {spinor }}^{\mu}$ is shown to be constant in time:

$$
\left(\Psi \mid \Psi^{\prime}\right)=\int d^{3} x k_{\text {spinor }}^{t}, \quad \frac{d}{d t}\left(\Psi \mid \Psi^{\prime}\right)=0,
$$

where the surface term should vanish:

$$
\begin{aligned}
& \left.k^{r}(x)\right|_{\substack{r=\infty \\
r=r_{\mathrm{H}}}} \\
= & \left.\frac{1}{\sqrt{\Delta_{\theta}}} \mathrm{e}^{-i\left(\omega-\omega^{\prime}\right) t} \mathrm{e}^{i\left(m-m^{\prime}\right) \phi}\left(R_{1, \lambda}^{*} R_{1, \lambda^{\prime}}-R_{2, \lambda}^{*} R_{2, \lambda^{\prime}}\right)\left(S_{1, \lambda}^{*} S_{1, \lambda^{\prime}}+S_{1, \lambda}^{*} S_{1, \lambda^{\prime}}\right)\right|_{r=r_{\mathrm{H}}} ^{r=\infty} \\
= & 0 .
\end{aligned}
$$

The boundary condition on $R_{1}, R_{2}$ are required for vanishing the surface term:

$$
R_{1}= \pm \gamma R_{2} \quad\left(r=r_{\mathrm{H}}\right), \quad \text { and } R_{1}, R_{2} \rightarrow 0 \quad(r \rightarrow \infty),
$$

where $\gamma$ is a phase factor.

The orthogonal relation for the separation parameter $\kappa$ is obtained by using the spinor equations and the boundary condition:

$$
\begin{aligned}
& \left(\Psi_{\omega, m, \kappa} \mid \Psi_{\omega, m, \kappa^{\prime}}\right) \\
= & i \int d^{3} x \frac{1}{\Xi}\left(\frac{r^{2}+a^{2}}{\Delta_{r} \sqrt{\Delta_{\theta}}}\left(R_{1, \lambda^{*}}^{*} R_{1, \lambda^{\prime}}+R_{2, \lambda^{2}}^{*} R_{2, \lambda^{\prime}}\right)+i \frac{a \sin \theta}{\sqrt{\Delta_{r} \Delta_{\theta}}}\left(R_{2, \lambda}^{*} R_{1, \lambda^{\prime}}-R_{1, \lambda}^{*} R_{2, \lambda^{\prime}}\right)\right)\left(S_{1, \lambda}^{*} S_{1, \lambda^{\prime}}+S_{2, \lambda}^{*} S_{2, \lambda^{\prime}}\right) \\
= & 0 .
\end{aligned}
$$

The orthonormal relations for the frequency $\omega$ and the azimuthal quantum number $m$ are obtained straightforward. Summarizing there results, orthonormal relations with respect to $\omega, m$ and $\kappa$ are obtained:

$$
\left(u_{\alpha} \mid u_{\alpha^{\prime}}\right)=-\left(v_{\alpha} \mid v_{\alpha^{\prime}}\right)=\delta_{\alpha, \alpha^{\prime}}, \quad\left(u_{\alpha} \mid v_{\alpha^{\prime}}\right)=\left(v_{\alpha} \mid u_{\alpha^{\prime}}\right)=0,
$$

with the eigenfunctions

$$
u_{\alpha}=\frac{\exp (-i \omega t+i m \phi)}{\left(\Delta_{r} \Delta_{\theta} \rho^{2} \sin ^{2} \theta\right)^{1 / 4}} F(r, \theta)_{\alpha}, \quad v_{\alpha}=u_{\alpha}^{\mathrm{c}}=i \gamma^{2} \gamma^{0} \bar{u}_{\alpha}^{T}
$$

where $\alpha=(\omega, m, \kappa)$ and $\alpha^{\prime}=\left(\omega^{\prime}, m^{\prime}, \kappa^{\prime}\right)$ denote sets of quantum numbers.

Any spinor fields can be expanded by eigenfunctions $u_{\alpha}$ and $v_{\alpha}$ :

$$
\Psi(t, x)=\Sigma_{\alpha}\left(c_{\alpha} u_{\alpha}+d_{\alpha}^{\dagger} v_{\alpha}\right),
$$

with the coefficient functions $c_{\alpha}=\left(u_{\alpha} \mid \Psi\right), d_{\alpha}^{\dagger}=-\left(v_{\alpha} \mid \Psi\right)$. Inserting the coefficient functions back into the spinor field expression, the completeness relations are obtained:

$$
i \sqrt{-g} \Sigma_{\alpha}\left(u_{\alpha}(t, x) \bar{u}_{\alpha}\left(t, x^{\prime}\right)-v_{\alpha}(t, x) \bar{v}_{\alpha}\left(t, x^{\prime}\right)\right) \gamma^{t}=\delta^{(3)}\left(x-x^{\prime}\right) .
$$




\section{Non-existence of zero mode and the spectrum condition}

The radial equations for zero mode, defining as $p_{\mathbf{H}}=\omega-\Omega_{\mathbf{H}} m=0$, become

$$
\begin{aligned}
& \left(\sqrt{\Delta_{r}} \partial_{r}-i \frac{\left(r^{2}-r_{\mathrm{H}}^{2}\right) \omega_{*}}{\sqrt{\Delta_{r}}}\right) R_{1 \mathrm{zero}}(r)=(\kappa-i \bar{\mu} r) R_{2 \mathrm{zero}}(r), \\
& \left(\sqrt{\Delta_{r}} \partial_{r}+i \frac{\left(r^{2}-r_{\mathrm{H}}^{2}\right) \omega_{*}}{\sqrt{\Delta_{r}}}\right) R_{2 \mathrm{zero}}(r)=(\kappa+i \bar{\mu} r) R_{1 \mathrm{zero}}(r),
\end{aligned}
$$

where $\omega_{*}=\Omega_{\mathrm{H}} m$ denotes the zero mode frequency. Near the horizon $\left(r=r_{\mathbf{H}}\right)$, zero mode equations become

$$
\sqrt{r-r_{\mathrm{H}}} \partial_{r} R_{1 \mathrm{zero}}(r) \simeq \beta R_{2 \mathrm{zero}}(r), \quad \sqrt{r-r_{\mathrm{H}}} \partial_{r} R_{2 \mathrm{zero}}(r) \simeq \beta^{*} R_{1 \mathrm{zero}}(r),
$$

where $\beta=\left(\kappa-i \bar{\mu} r_{\mathrm{H}}\right) / \sqrt{X\left(r_{\mathrm{H}}\right)}$ with $X(r)=\partial_{r} \Delta_{r}$.

General solutions to eq.(18) are given by

$$
\begin{aligned}
& R_{1 \text { zero }}(r) \simeq d_{1} \exp \left(2|\beta| \sqrt{r-r_{\mathrm{H}}}\right)+d_{2} \exp \left(-2|\beta| \sqrt{r-r_{\mathrm{H}}}\right) \\
& R_{2 \mathrm{zero}}(r) \simeq\left(\beta^{*} / \beta\right)^{1 / 2}\left(d_{1} \exp \left(2|\beta| \sqrt{r-r_{\mathrm{H}}}\right)-d_{2} \exp \left(-2|\beta| \sqrt{r-r_{\mathrm{H}}}\right)\right)
\end{aligned}
$$

where $d_{1}$ and $d_{2}$ are integration constants. For the boundary condition

$$
R_{1}=\left(\beta / \beta^{*}\right)^{1 / 2} R_{2}, \quad \text { or } \quad R_{1}=-\left(\beta / \beta^{*}\right)^{1 / 2} R_{2},
$$

where the phase factor is $\beta=\left(\kappa-i \bar{\mu} r_{\mathrm{H}}\right) / \sqrt{X\left(r_{\mathrm{H}}\right)}$ with $X(r)=\partial_{r} \Delta_{r}$, zero mode solutions cannot satisfy the boundary condition in eq.(20). This means that the zero mode does not exist as physical states,i.e. "non-existence of zero mode".

Next we give the statement on the spectrum in the following.

(1) In case without rotation ( $a=J / M=0)$, the physical modes satisfy the condition $\omega>0$.

(2) In case with rotation $(a=J / M \neq 0)$, any mode cannot across the zero mode line $\left(\omega-\Omega_{\mathrm{H}} m=0\right)$.

(3) The analyticity with respect to the rotation parameter $a$ is required under the conditions:

(i) the well-separated zero of $\Delta_{r}$ as outer horizon $\left(r_{\mathbf{H}}\right)$ and

(ii) the well-defined value of $\Delta_{\theta}$ as $a<\ell$ (smaller value of rotation parameter than cosmological parameter).

Then the set of quantum number $\alpha=(\omega, m, \kappa)$ should satisfy the spectrum condition $\omega-\Omega_{\mathrm{H}} m>0$.

\section{Superradiant modes}

General superradiant condition is derived under the conditions (a) the area law of BH and (b) the conservation law of energy and angular momentum by Bekenstein [2] and others [3, 4] as

$$
1-\frac{\Omega_{\mathbf{H}} m}{\omega}<0 \text { or } \omega\left(\omega-\Omega_{\mathbf{H}} m\right)<0,
$$

from which there are two possible types of superradiance:

type 1 superradiance: $\omega>0$ and $\omega-\Omega_{\mathbf{H}} m<0$ or type 2 superradiance: $\omega<0$ and $\omega-\Omega_{\mathbf{H}} m>0$.

Our spectrum condition $\omega-\Omega_{\mathbf{H}} m>0$, derived in sect. 4 , , requires that type 1 superradiance is not consistent and type 2 superradiance is consistent to occur as stable superradiant modes. 


\section{Summary}

As the summary of this report, we classify the superradiant modes as follows. ( $p_{\mathbf{H}} \equiv \omega-\Omega_{\mathbf{H}} m$ denotes the momentum of matter fields on the horizon):

For spinor fields,

type $1\left(\omega>0\right.$ and $\left.p_{\mathbf{H}}<0\right)$ is not possible but type $2\left(\omega<0\right.$ and $\left.p_{\mathbf{H}}>0\right)$ is possible by our analysis. (Note that both types are not possible by other analysis $[3,5,6]$.)

For scalar fields, which is described in our paper [1] though not in this report, type $1\left(\omega>0\right.$ and $\left.p_{\mathbf{H}}<0\right)$ is not possible but type $2\left(\omega<0\right.$ and $\left.p_{\mathbf{H}}>0\right)$ is possible by our analysis. (Note that the results are opposite by other analysis. )

The result of our analysis is obtained from the completeness relations in sect. 3 and the spectrum condition in sect.4. For spinor and scalar fields, one of superradiance should be possible, because of the completeness relation. Among two types, type 2 can occur because of the spectrum condition. We have obtained the same type 2 superradiance both for spinors and scalars, which is supported by Bargmann-Wigner formulation by our previous work [7].

\section{References}

[1] M. Kenmoku, Y. M. Cho, K. Shigemoto and J. H. Yoon, arXiv:1609.00798, to appear in Classical and Quantum Gravity.

[2] J. D. Bekenstein, Phys. Rev. D7, 949 (1973).

[3] W. G. Unruh, Phys. Rev. Lett. 31, 1265 (1973), Phys. Rev. D10, 3194 (1974).

[4] S. Mano and E. Takasugi, Prog. Theor. Phys. 97, 213 (1997).

[5] S. Meda, Prog. Theor. Phys. 55, 1677 (1976).

[6] S. M. Wagh and N. Dadhich, Phys. Rev. D32, 1863 (1985).

[7] M. Kenmoku and Y. M. Cho, Int. J. Mod. Phys. A30, 1550052 (2015). 